\title{
A Camouflaged Case of Disseminated Tuberculosis Presenting as Adrenal Crisis
}

\author{
Saquib Navid Siddiqui
}

\section{ABSTRACT}

Lymphohematogenous spread of mycobacterium tuberculosis to multiple organs presents a complex diagnostic challenge to any physician. A holistic and vigilant approach is required in the quest to diagnose disseminated tuberculosis causing adrenal failure. Although tuberculosis can affect various endocrine glands of the body yet adrenal remains the most common.[1] It is also the fifth most common site for extra-pulmonary tuberculosis.[2] The incidence of tuberculous Addison's disease has lowered courtesy of anti-tubercular medications accounting for only $7-20 \%$ of cases.[1] Here we showcase a rare incidence where a 39 years old gentleman with no constitutional symptoms and no known co-morbidities presenting with neck pain went into adrenal crisis and eventually was found to be due to disseminated tuberculosis which affected his lungs, adrenal glands, cervical spine, and brain.

Keywords: Disseminated tuberculosis, tubercular Addison's disease, antitubercular therapy with steroid, rifampicin and steroid drug interaction.
Submitted : April 17, 2021

Published : May 10, 2021

ISSN: $2593-8339$

DOI: $10.24018 /$ ejmed.2021.3.3.819

Dr Saquib Navid Siddiqui *

Specialist Registrar, Respiratory Medicine \& General Medicine, Northumbria Healthcare NHS Foundation Trust, UK.

(e-mail: sns.saquib@gmail.com)

*Corresponding Author

\section{INTRODUCTION}

Primary adrenal failure is a life-threatening medical emergency that requires immediate attention. We present a case of a 65-year-old gentleman with no previously known co-morbidities presenting to the emergency unit with a week's history of neck pain and dizziness. Initially, he was admitted under the orthopedic team but later during the course of hospital admission, collapsed in the hospital leading to the involvement of the medical team. Biochemical tests confirmed primary adrenal failure. Subsequent chest imaging showed miliary mottling and a suprarenal $\mathrm{CT}$ with contrast demonstrated bi-lateral adrenal masses which was later confirmed by CT guided FNAC to have appearances consistent with adrenal tuberculosis. Spine and brain imaging tests found the presence of tuberculoma. He was treated with simultaneous anti-tubercular therapy and adrenal hormone replacement therapy.

\section{CASE Presentation}

A 65-years-old gentleman with no known co-morbidities presented to the emergency unit with a one-week history of neck pain and dizziness. Initially, he was assessed by the orthopedic team and was admitted under their care for further workup as an inpatient. During the course of the admission, he collapsed whilst walking to the toilet, necessitating the need for the involvement of the medical team to assess the cause of the fall. On examination, he was found to be hypotensive with an initial BP of $85 / 55 \mathrm{mmhg}$ with a significant postural drop. Further delving into the history, it was revealed that he has been running low blood pressure for the past few months and had been drinking the extra amount of water and saline to help with it. Laboratory investigations showed a sodium level of $120 \mathrm{~m} . \mathrm{mol} / \mathrm{l}$ with normal potassium (4.8 m.mol/l). In view of a possible adrenal crisis, an immediate blood sample was taken to measure the random cortisol level, and soon after, intravenous hydrocortisone and fluids were administered with dramatic clinical improvement. As the cortisol level came back $2.2 \mathrm{mcg} / \mathrm{dl}$ an early morning short synacthen test was requested withholding the morning dose of steroid which confirmed the diagnosis of primary adrenal failure. Routine x-ray chest and later HRCT chest (Fig. 1) showed miliary mottling. Further history revealed exposure to tuberculosis patient from his same household 15 years ago. In conjunction with the adrenal crisis and the military mottling, disseminated tuberculosis affecting adrenal glands was added to the differential diagnosis which was confirmed with a report of bilateral adrenal mass in a suprarenal CT with contrast (right adrenal mass about $5.5 \times 5.0 \times 3 \mathrm{~cm}$ and left adrenal mass about $6.5 \times 4.7 \times 3.7 \mathrm{~cm}$ ) (Fig. 1). An MRI cervical spine showed spinal tuberculoma with the paravertebral lesion (Fig. 2) and an MRI brain revealed cerebral tuberculoma (Fig. 2). CT guided FNAC of the adrenal glands revealed epithelioid granulomas with the multinucleated giant cells with background necrosis. He was tested for HIV which was negative. 


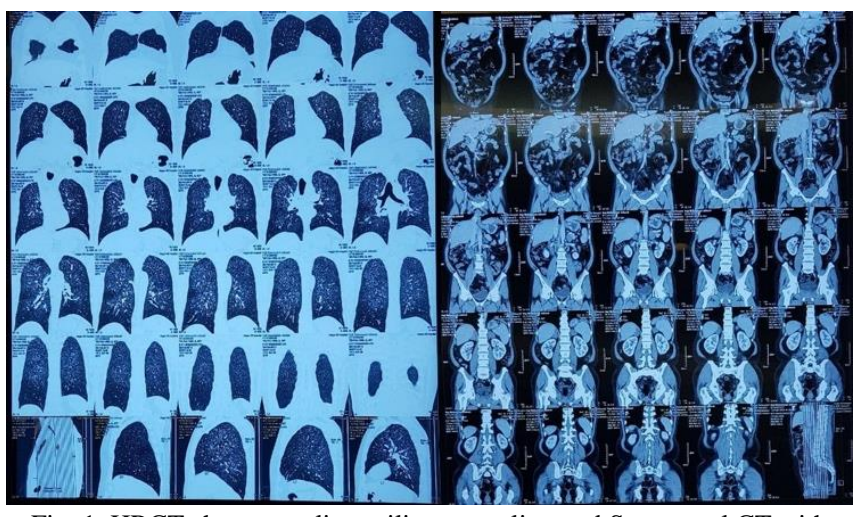

Fig. 1. HRCT chest revealing military mottling and Suprarenal CT with contrast showed the presence of bilateral adrenal masses (right adrenal mass about $5.5 \times 5.0 \times 3 \mathrm{~cm}$ and left adrenal mass about $6.5 \times 4.7 \times 3.7 \mathrm{~cm}$ ) with an enlarged left para-aortic lymph node.

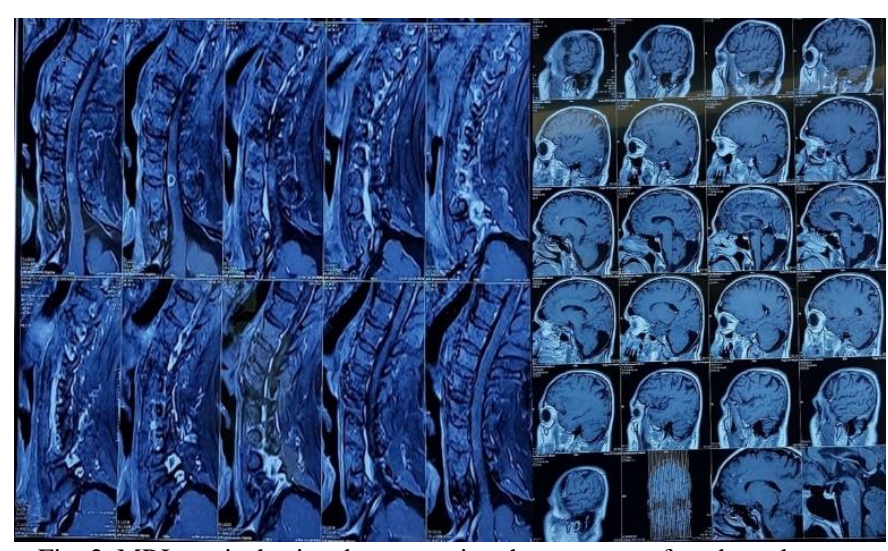

Fig. 2. MRI cervical spine demonstrating the presence of a tuberculoma along with a paraspinal lesion and MRI brain revealed multiple rim enhancing 'target lesions' of variable sizes scattered in both cerebral hemispheres.

Lab results revealed euvolemic hypo-osmolar hypernatriuric hyponatremia with serum sodium of 120 $\mathrm{mmol} / \mathrm{L}$ and osmolality $262 \mathrm{mOsm} / \mathrm{L}(280-296 \mathrm{mOsm} / \mathrm{L})$, urine sodium $148 \mathrm{mmol} / \mathrm{L}$, and osmolality $510 \mathrm{mmol} / \mathrm{L}$ (300$900 \mathrm{mmol} / \mathrm{L}$ ). Inflammatory markers were elevated i.e., Creactive protein was $157 \mathrm{mg} / \mathrm{dL}$ with an ESR of $132 \mathrm{~mm}$ in the 1st hour. Random cortisol levels from the blood sample taken prior to steroid administration was $2.2 \mathrm{mcg} / \mathrm{dL}$. Subsequent ACTH stimulation test (short synacthen test) performed on the following morning with holding morning dose of steroid revealed serum cortisol of $3.4 \mathrm{mcg} / \mathrm{dL}$ on 0 minutes and the 30mins cortisol value was $1.9 \mathrm{mcg} / \mathrm{dL}$ confirming adrenal insufficiency. ACTH and aldosterone levels on 0 minutes were $1130.8 \mathrm{pg} / \mathrm{mL}(6-48 \mathrm{pg} / \mathrm{mL})$ and below $1.1 \mathrm{ng} / \mathrm{dL}$ respectively confirming the etiology of adrenal failure to be primary in origin. Tuberculin test revealed an induration of $30 \mathrm{~mm}$ (patient had BCG vaccine). Blood MTB PCR and sputum culture was positive for mycobacterium tuberculosis sensitive to both isoniazid and rifampicin.

$\mathrm{He}$ was commenced on intravenous hydrocortisone (100 mg stat followed by $50 \mathrm{mg}$ four times daily) with intravenous fluids as soon as initial blood samples were taken to measure basal cortisol. Later after confirmation of primary adrenal failure, he was started on adrenal hormonal replacement in the form of oral hydrocortisone and fludrocortisone. Initially, he was prescribed $20 \mathrm{mg} /$ day oral hydrocortisone (15 mg-5 mg after each meal) along with antitubercular therapy as he was found to have disseminated tuberculosis. Later due to relatively slow clinical response, his daily dose was increased to $30 \mathrm{mg}$ (10 mg-10 mg-10 mg after each meal). After which he showed dramatic clinical improvements. Once he was commenced on oral hydrocortisone and was eating and drinking adequately oral fludrocortisone was added at the dose of $100 \mathrm{mcg} /$ day which was later increased to $150 \mathrm{mcg} /$ day after which his sodium level in the blood raised and remained within normal limits throughout the course of his hospital stay. Oral potassium supplementation was added to prevent fludrocortisone related hypokalemia. His initial 2 months of anti-tubercular therapy comprised of isoniazid, rifampicin, ethambutol, and pyrazinamide, followed by 12 months of extended therapy with isoniazid and rifampicin only, and after stopping his anti-tubercular therapy we reduced his dosage of hydrocortisone to $20 \mathrm{mg}$ /day ( $15 \mathrm{mg}-5 \mathrm{mg}$ after each meal). He was given cervical orthosis as advised by the orthopedic team along with analgesics for neck pain. Prophylactic antiepileptic medication for ring-enhancing lesions of the brain was not initiated as per neurology advice till the onset of any seizure-like activity which later never required as his symptoms improved without developing any neurological features.

\section{DISCUSSION}

Bloodborne dissemination of tuberculosis to two or more organs is known as dissemination tuberculosis, which is found in only $2-5 \%$ of patients with tuberculosis [5]. The presence of a high concentration of corticosteroid and rich vascularity makes adrenal glands an easier target for tuberculosis [2]. Although autoimmune destruction of the adrenal gland is the commonest cause of Addison's disease in the western world, tuberculosis remains the most common cause of primary adrenal failure in developing countries, with an incidence rate of 7-20\% [2]. Tuberculosis of the adrenal gland results in the formation of granulomas, inflammation, necrosis, calcification, and destruction of the adrenal cortex [2], [6]. Clinical features largely depend upon loss of hormonal functions. Loss of cortisol results in fatigue, tiredness, nausea, vomiting, abdominal pain, hypoglycemia, hypercalcemia. Loss of aldosterone function results in postural hypotension and electrolyte changes (hyponatremia in $80 \%$ cases and hyperkalemia in $40 \%$ cases) [2], [7].

The deficiency of androgen also causes problems in secondary sexual characteristics. Loss of adrenal function stimulates the release of ACTH from the anterior pituitary and excessive secretion results in skin pigmentation over the sun-exposed areas of the body [2]. Spinal tuberculosis commonly affects the lower thoracic and lumbar spine, barely $2-3 \%$ of cases are reported with cervical spine involvement [8]. It can result in progressive worsening in neurological deficits including paraplegia and kyphotic deformity of the cervical spine [3]. Spreading into the brain can lead to inflammation of the meninges, formation of tuberculoma, and brain abscess. Around 33\% of space-occupying lesions are due to tuberculosis [4].

Smear and culture of sputum for acid-fast bacilli along with nucleic acid amplification testing remains a cornerstone for diagnosing tuberculosis [5]. Other options are interferongamma release assay (quantiferon gold test), tuberculin test, 
bronchoscopic sampling. Extra-pulmonary tuberculosis sites are sampled for acid-fast bacilli smear, mycobacterial culture, nucleic acid amplification testing, and histological examination [5]. Various biochemical tests are required to diagnose Addison's disease namely, the ACTH stimulation test (short synacthen test) confirms the diagnosis whereas baseline measurement of ACTH, aldosterone levels will aid in differentiating primary and secondary/tertiary causes.[7] Appropriate imaging will be required to detect adrenal, spinal, and brain tubercular lesions. Usual imaging finding in the case of adrenal tuberculosis is bilateral enlarged adrenal glands with or without calcification [2], [6]. Usually, calcifications and gland atrophy are commonly found in chronic tubercular adrenal disease [2], [6].

Anti-tubercular therapy is indicated in all forms of extrapulmonary tuberculosis. Therapy includes two months of the intensive phase with four medications namely isoniazid, rifampicin, ethambutol, pyrazinamide followed by a continuation phase with dual anti-tubercular drugs i.e., isoniazid and rifampicin [5].

Addison's disease is mainly treated in the acute crisis phase by intravenous steroids and fluids which is followed by replacement of the deficient hormones usually hydrocortisone, fludrocortisone, and androgens [7]. Very few patients are known to recover complete adrenal function following completion of anti-tubercular therapy in Addison's disease secondary to tuberculosis particularly if the gland develops calcification and becomes atrophic [8], [9]. Recovery relies on the amount of adrenal tissue destruction caused by tuberculosis and the presence of viable tissue at the time of diagnosis [10].

Anti-tubercular drugs are also not required if adrenal glands are atrophic and calcified as recovery is unlikely [8], [10]. Rifampicin has been previously reported to shorten the half-life of steroids along with impairment of the therapeutic response as it is a strong inducer of the cytochrome $\mathrm{P} 450$ (CYP) system which is involved in the metabolism of adrenocorticoids. Although currently no guidelines are available for the dose adjustments, it is advisable to adjust the dose as per clinical and biochemical response when the enzyme inducer is initiated and stopped [11].

\section{CONCLUSION \& LEARNING POINTS}

- Combination of anti-tubercular medications along with hormone replacement therapy can be life-saving in tubercular Addison's disease.

- Steroidal dose adjustment might be required in case of tubercular Addison's disease due to simultaneous use of rifampicin which acts as cytochrome P450 inducer.

\section{ACKNOWLEDGMENT}

I thank our patient to give us permission to tell his story for the benefit of medical science.

\section{REFERENCES}

[1] Ali-Mamari A., Balkhair A., Gujjar A., et al. A Case of Disseminated Tuberculosis with Adrenal Insufficiency. PMID: 21509318; PMCID: PMC3074785.

[2] Manso M. C., Rodeia S. C., Rodrigues S., et al. Synchronous presentation of two rare forms of extrapulmonary tuberculosis. 2016 Apr 18; doi: 10.1135/bcr-2015-212917.

[3] Sadek A.-R., Wallage W., Jaiganesh T. Cervical spine tuberculosis causing instability and neurological compromise. 2011 Jun 6; doi: 10.1258/shorts.2011.011040.

[4] Saleh M, Saeedi A. A., Pooran A. A. Brain Tuberculomas: A Case Report. 2014 Jul;7(7):e11252; doi: 10.5812/jjm.11252.

[5] Chamberlin K., Orfanos S., Mukherjee A., et al. A case of disseminated tuberculosis mimicking metastatic cancer. 2018 Oct 2; doi: 10.1016/j.rmcr.2018.10.001.

[6] Arambewela M., Ross R., Pirzada O., et al. Tuberculosis as a differential for bilateral adrenal masses in the UK. 2019;12:e228532; doi:10.1136/bcr-2018-228532.

[7] Puttanna A., Cunningham A. R., Dainty P. Addison's disease and its associations. July 20, 2013; doi: 10.1136/bcr-2013-010473.

[8] Kelestimur F. Recovery of adrenocortical function following treatment of tuberculous Addison's disease. 01 Oct 1993; doi: 10.1136/pgmj.69.816.832.

[9] Bhatia E., Jain S. K., Gupta R. K., et al. Tuberculous Addison's disease: lack of normalization of adrenocortical function after anti-tuberculous chemotherapy. 25 December 2001; doi: 10.1046/j.13652265.1998.00409.x.

[10] Trianto H., Betty N., Sasiarini L., Rosandi R., et al. Acute Adrenal Insufficiency as the Primary Manifestation of Extrapulmonary Tuberculosis: A Case report. May 25,2016; doi: 10.15605/jafes.031.01.11.

[11] Park B. K., Breckenridge A. M. Clinical implications of enzyme induction and enzyme inhibition. PMID: 6113907; doi: 10.2165/00003088-198106010-00001.

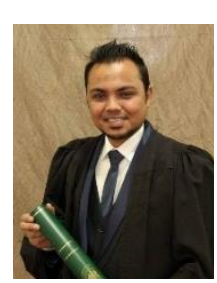

Dr. Saquib Navid Siddiqui completed MBBS in 2012 in Dhaka, Bangladesh. During his MBBS journey he obtained highest marks in all 3 professional examinations from his medical college remaining within top $15 \%$ of the total marks. Since then, he underwent training in various sub-specialties of medicine in various tertiary level hospitals in Bangladesh. He completed MRCP (UK) in 2016 (One of the top scorer in MRCP paces exams from Bangladesh) and joined NHS in 2019 as trust grade specialist registrar in general medicine. He is currently working as a higher speciality trainee in respiratory medicine \& general medicine. He is the recipient of 3 prestigious awards while working for NHS (Covid 19 recognition award, Epic award, and Covid-19 appreciation award)

He has 5 other publications as the lead and corresponding author to his credit:

1. 'A Unique Tale of Covid-19 induced concomitant overt disseminated intravascular coagulation and acute bilateral pulmonary embolism' published in International Journal of Infectious Diseases on January, 2021.

2. 'Is standard Oral Dose Dexamethasone (6mg Once Daily) Prescribed for Covid-19 Pneumonitis Treating Autoimmune Haemolytic Anaemia Associated with Covid-19?

3. A case Series of Five Patients Providing Us with the answer' published in IJIRMS on January 2021.

4. 'A Rare Case of Alcohol Intoxication Masquerading Cerebral Venous Sinus Thrombosis' published in EJMED on 20/11/2020.

5. 'A Case of Massive Pulmonary Embolism in Covid-19 Pneumonitis' published in IOSR journal on 19/06/2020.

6. 'A Diagnostic Road to Damascus: A Case of Conversion to Pontine Infarct' published in IOSR journal on 10/07/2020.

$\mathrm{He}$ is an active contributor in providing education to the junior doctors of his trust by regularly participating in delivering various teaching sessions. $\mathrm{He}$ is also the initiator of a weekly teaching session in his previous trust (EKHUFT) in elderly care. He has completed formal course in teaching to improve his teaching skills. He has completed two qualitive improvement projects in his previous trust (EKHUFT) which has had profound influence in improving quality of providing health care service in the respective area (Quality improvement project on 'Evidence based clinical guidelines for the management of Covid-19 on the Oxford Ward High Dependency Unit' in 2020 and Quality improvement project on 'Prescription of Therapeutic Oxygen as Drug' in 2020). He has had 3 poster presentations in Society of Acute Medicine conference held in Glasgow, 2020. He has participated and completed various clinical courses and skill development programmes since 
starting his career in NHS 2019. In addition, he is actively working as associate regional advisor for the Royal College of Physicians of Edinburgh since May 2020. He is the lead author of Guidance of use of CPAP in Covid19 Pneumonitis which was accepted by the Gold command of his previous trust (EKHUFT) to be used in all patients with hypoxic respiratory failure along with covid-19 pneumonitis requiring CPAP support. He is a member of the British Thorasic Society, European Respiratory Society and American College of Chest Physician. 